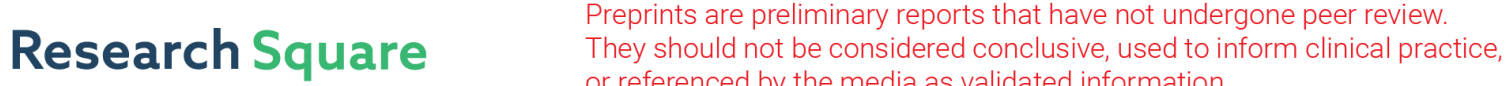 or referenced by the media as validated information. \\ Analysis of Industry Convergence Based on Improved Neural Network
}

Nan Ma ( $\sim$ clear51688@163.com )

Fuzhou University of International Studies and Trade

\section{Research Article}

Keywords: Neural network, improved algorithm, industry convergence, intelligent model

Posted Date: September 8th, 2021

DOI: https://doi.org/10.21203/rs.3.rs-849560/v1

License: (9) This work is licensed under a Creative Commons Attribution 4.0 International License. Read Full License

Version of Record: A version of this preprint was published at Soft Computing on December 3rd, 2021. See the published version at https://doi.org/10.1007/s00500-021-06439-0. 


\title{
Analysis of industry convergence based on improved neural network
}

\author{
Nan Ma \\ Fuzhou University of International Studies and Trade, Fuzhou, Fujian, China,350202 \\ Email: clear51688@163.com
}

\begin{abstract}
Economic growth in the information age is no longer a stage driven by unipolarity. It has entered a multi-polar driving stage characterized by integration, fusion, and integrated development on a larger scale between regions, and the trend of group competition with urban agglomerations as carriers has become increasingly obvious. This paper improves the neural network algorithm based on the needs of industrial economic integration in the digital age, and proposes an industry convergence analysis model based on the improved neural network algorithm. Moreover, this article combines industry models to analyze actual needs and constructs an industry convergence analysis model based on improved neural networks, and analyzes the integration of different industries. In addition, this article conducts experiments through multiple sets of data, and combines the neural network model of this article to conduct research. Through experimental research, we know that the model constructed in this paper can play an important role in the analysis of industry convergence.
\end{abstract}

Keywords: Neural network; improved algorithm; industry convergence; intelligent model

\section{Introduction}

As a brand-new industrial organization and development model, industry convergence has shown a vigorous development trend in various countries around the world, and it has brought a new development direction to the development of the global industrial economy.Industry convergence is an important way to promote regional economic development. Through the effective combination of points, axes, planes and other spatial elements between regions, the continuous adjustment and optimization of the regional industrial structure is guided to form a multi-level gradient energy difference, thereby creating conditions for industrial transfer. The gradient or jump transfer of regional industries can strengthen the cooperation between industrial groups, and then form an industrial interest community, thereby inducing industrial gradient upgrades in various regions [1].At present, the phenomenon of China's industry convergence continues to emerge and the trend continues to increase. In particular, the phenomenon of industry convergence between regions is becoming more and more active. China's regional development strategy has shifted from priority development to unbalanced development in coastal areas to the comprehensive and coordinated development of northeast, west, and central regions [2].With the implementation of the strategy for the rise of the central region, the construction of urban clusters in the central region, especially the middle reaches of the Yangtze River, is on the agenda. In 2010, the "National Plan for Major Functional Zones" promulgated by the State Council marked the rise of the middle reaches of the Yangtze River, which is composed of the three main bodies of Wuhan City Circle, Changsha-Zhutan City Group, and Poyang Lake Eco-economic Zone, as a key development area at the national level.At the same time, the "Twelfth Five-Year Plan Outline" pointed out to accelerate the construction of urban clusters in the middle reaches of the Yangtze River with Wuhan, Changsha, and Nanchang as the core. In February 2012, the provincial governments of Hubei, Hunan, and Jiangxi signed the "Agreement on Accelerating the Construction of Strategic Cooperation Framework for Urban Clusters in the Middle Reaches of the Yangtze River", which indicates that the construction of urban agglomerations in the middle reaches of the Yangtze River has taken a step forward.On April 5, 2015, the Chinese government approved the "Development Plan for the Urban Agglomeration in the Middle Reaches of the Yangtze River". This is an important measure to implement the major national strategy of the Yangtze River Economic Belt, and it is also the first cross-regional urban agglomeration plan approved by the state after the "National New Urbanization Plan (2014-2020)" was issued. This is of great significance for accelerating the overall rise of the central region, exploring new urbanization paths, and promoting the development of regional integration. The foundation of industrial development and the dependence of spatial economic interests have created good preconditions for the development of regional industry convergence [3].

As the most important carrier and realization form of the regional economy, the unique advantages of urban agglomerations make it possible for the emergence and realization of industrial integration. At present, China's economic growth is no longer a unipolar-driven stage. It has entered a multi-polarity-driven stage characterized by regional integration, integration, and integrated development on a larger scale, with urban agglomerations as the carrier. The trend of group competition is becoming increasingly obvious.

Throughout the history of the world's economic and social development, every industrial technological revolution promotes industrial development through technological innovation and creates new leading industries. Through the penetration and application of leading industries, the transformation and upgrading of traditional industries is promoted, which has a huge impact on human activities. It has a profound impact and continuously improves the level of human understanding of the world and the ability to transform the world. Since the beginning of the 21 st century, digital technology has developed rapidly, and has been widely penetrated and applied in various industrial fields, accelerating the reconstruction of the material foundation of the economy and society, and the development of human economy and society has also entered a new stage. On the one hand, the leading sector with the basic industries of the digital economy as the core realizes industrialization; on the other hand, it causes the digital trans- 
formation of industries in other industrial fields of the economy and society. The digital technology revolution also follows Schumpeter's "disruptive innovation" concept, which will cause a series of changes in enterprises, industries, and macroeconomics. In view of this, considering the comprehensive influence of the digital economy on various fields of the economy, the Economist Intelligence Unit proposes that the spread of digital technology from the preparatory stage to the spreading stage of the digital economy. Therefore, under the background of general digital technology, network infrastructure, economic globalization and technological economic order, it will go through the evolutionary process of technological innovation, industrial restructuring, integrated application, and institutional transformation. The economy came into being.

This paper combines the improved neural network algorithm to construct the industry convergence analysis model, analyzes the industry convergence, improves the effect of industry convergence strategy formulation, and improves the reliability of industry convergence.

\section{Related work}

The literature [4] boldly proposed that industry convergence is based on digital integration through the study of the evolution of the machinery industry.The literature [5] pointed out that in the era of experiential consumption, operators need to win consumers by improving product quality, adopt cultural and creative means, guide tourists, and generate new economic value.The literature [6] believed that a variety of different personal travel behavior needs lead to the integration of heritage and tourism. The literature [7] believed that there are five dimensions of integration: equipment, original technology, network, enterprise and regulation.The literature [8] emphasized agricultural diversification, reduces farmers' sole reliance on traditional agriculture, changes their identity, and can be used as shop assistants or provide leisure. The literature [9] defined four major forms through the theory of economics and life cycle theory, establishes industry convergence, and forms a theoretical framework of dynamic evolution. The literature [10] believed that cultural tourism should be self-learning, which can promote personal improvement and promote economic development. The literature [11] used life cycle theory model deduction, emphasizing that technological innovation can define the boundary of industry convergence.The literature [12] took South Africa as the research object to point out that cultural creativity promotes the development of urban tourism. The literature [13] analyzed the industrial CBTI model based on the stakeholders of rural tourism benefits.

Literature [14] uses the entropy method and the coupling degree model to propose the need to strengthen the comprehensive development of industries, improve market cultivation, and optimize the development of inter-city industries. Literature [15] emphasizes the strategies of scientific preparation and planning of industrial integration, strengthening regional linkage, creating large-scale folk tourism performances, establishing policy guarantees, and improving talent mechanisms. Literature [16] analyzes the motives of the integration of the cultural industry and the tourism industry, which mainly lie in the market demand, the enterprise's pursuit of maximum efficiency, technological innovation, and the relaxation of management mechanisms. The integration of industries has given birth to new products, markets, and enhancements. The added value of the industrial chain, the strengthening of organization and management innovation, and the extension of the life cycle. Literature [17] established a dynamic model for the integration of cultural industry and tourism industry from the four forces: push, pull, support, and resistance, and proposed four integration paths: integration, absorption, reorganization, and penetration and integration. The literature [18] uses the coupling coordination degree to measure and shows that the regional economy needs to strengthen the technical integration of cultural resources, the region needs to strengthen the technical integration of tourism resources, and strengthen the path of market integration of tourism resources. Literature [19] uses the Lotka-Volterra model to calculate, and the result analysis is already in a state of integration, but the innovation is insufficient, the brand awareness is not high, and the deep integration needs to be improved. Literature [20] pointed out that the cultural industry and the tourism industry should be promoted at the same time at the macro and micro levels. Literature [21] believes that the promotion of the integration of cultural industry and tourism industry presents a positive dynamic inertia, and shows a trend of convergence; among them, consumer demand, technological innovation, and government regulations are the basic driving forces to promote the integration of cultural tourism industry. Literature [22] summarizes the development model of the digital economy through the combined effect of the additive effect and the multiplicative effect, and cultivates a new business form that integrates the development of the three major industries in the countryside. Literature [23] used the coupling coordination degree model to measure the integration level of multiple regional cultural tourism industries, and it turns out that the combined development of the two industries can drive the level of cultural consumption.

\section{Improved neural network algorithm}

With the improvement of computing performance and the development of artificial intelligence technology, artificial neural networks have become popular. The construction of the artificial neural network model draws on human neurons. A neuron is a node in the network, and different connection methods between neurons can construct a wide range of different network models. In the computer field, this kind of network is collectively called a neural network, which is usually a calculation model constructed using a large number of neuron nodes.

Generally speaking, a four-layer artificial neural network is shown in Figure 1. The network includes an input layer $X_{1}, X_{2}, X_{3}, X_{4}$

represents input features), two hidden layers, and an output layer (y represents output).A neural network is composed of a set of connected units called nodes or neurons, and it is like biological neurons in the brain. These neurons integrate input signals and transmit them to other connected neurons. The output of the neuron is the weighted sum of the neurons in the previous layer. However, this neural network model is prone to translation and displacement distortion, which leads to poor classification results. 


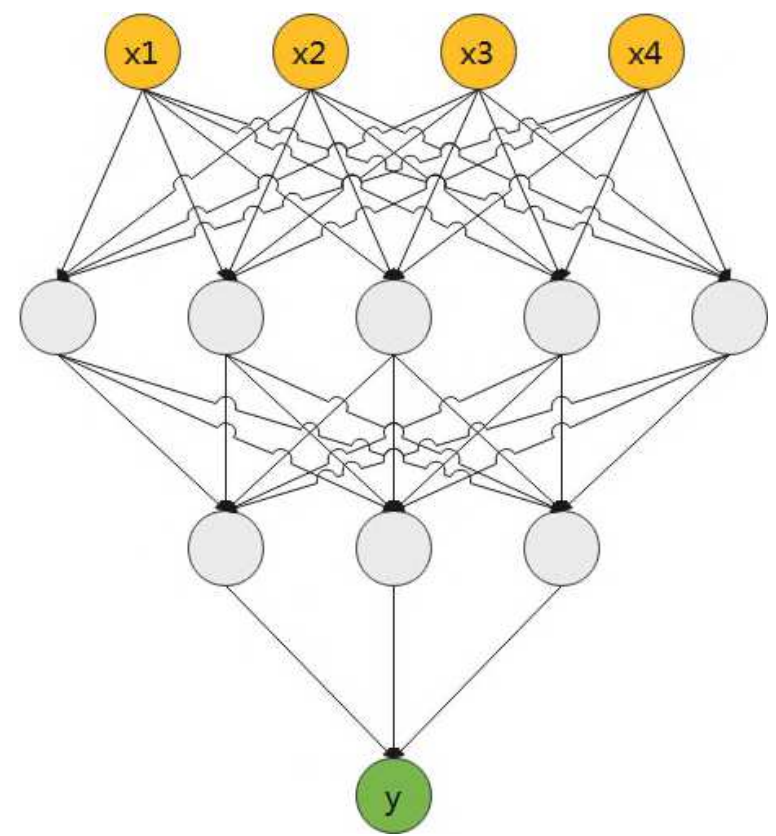

Figure 1 Artificial neural network

The modern convolutional neural network is developed on the basis of the early convolutional neural network model represented by the LeNet-5 model. With the improvement of computer hardware and computing performance, in order to improve the fitting ability of the model, the number of layers of the network is gradually increasing, and the computing performance is also required to be higher, hence the name of the deep learning technology. Since then, with the continuous emergence of deep learning theories, data blowout generation, and substantial improvements in computing equipment, convolutional neural networks have developed rapidly and have been applied to computer vision, natural language processing, and of course, they are also widely used in biology. Fields and other fields.

The convolutional neural network algorithm is based on artificial neural networks, and some improvements have been made on it. These improvements overcome the shortcomings of artificial neural networks. The convolutional layer is used after the input layer and pooling is used after the convolutional layer. Floor. Usually, multi-layer convolutional neural networks are used to extract the pain EEG information of low back pain. This feature is relatively abstract. Multi-layer convolution can enhance the signal while reducing noise.

Convolutional layer: A large number of operations are convolutional operations. Usually this layer of network is also called a feature extraction layer. It uses local connections and weight sharing to extract the primary features of low back pain pain EEG signals. The most important thing in the convolutional layer is the convolution operation. If a two-dimensional grid data (usually an array) is used as the input of the convolution operation (its identification is I, the coordinates are ( $\mathrm{m}, \mathrm{n}$ )), the convolution kernel should also correspond to a two-dimensional grid data (Its identification is K), of course, the obtained feature map is also a twodimensional grid data (its identification is $S$, coordinates are $(i, j)$ ).

The process of convolution operation can use the following formula as shown in (1): $S(i, j)=\left(\mathrm{I}^{*} K\right)(i, j)=\sum_{m} \sum_{n} I(m, n) K(i-m, j-n)$

This is a process in which the corresponding data is multiplied and the final product is summed. For a convolution operation, the input and the convolution kernel are interchangeable, so the equivalent formula is shown in (2):

$$
S(i, j)=(K * \mathrm{I})(i, j)=\sum_{m} \sum_{n} I(i-m, \mathrm{j}-n) K(m, n)
$$

In practical applications, for simplicity, usually the convolution operation is implemented in many machine learning libraries as the following calculation formula as shown in formula (3):

$$
S(i, j)=(K * \mathrm{I})(i, j)=\sum_{m} \sum_{n} I(i+m, \mathrm{j}+n) K(m, n)
$$

For practical application considerations, the convolution operation used in the future will be implemented in this form, and it will also be assumed that it is a convolution operation. Generally, I is a two-dimensional matrix composed of data values, and $\mathrm{K}$ is a weight parameter optimized by a learning algorithm. It is also a two-dimensional matrix (or more).

Pooling layer: It is also called down-sampling layer. The role of the pooling layer is to reduce the dimensionality of features extracted by the convolutional layer. The features extracted by the tape measure layer have the problem of large dimensions. If they are not processed and directly input to the network, a huge amount of calculation will be generated, which may lead to poor classification results.Therefore, the classic neural network algorithm will use the pooling layer to reduce the feature dimension after 1-2 layers of convolution operation, and then input to the full connection or input to the convolution layer to continue to extract the features, which can greatly improve the efficiency.

Sparse connection and parameter sharing are two important characteristics of convolution operation, and the neural network model can be improved through these characteristics. Below, we briefly introduce these two features.

(1) Sparse connection. Convolution operation has the characteristics of sparse connection or sparse interaction. The schematic diagram of sparse connection and full connection is shown in Figure 2. The input unit is expressed in the form of xi, and the output unit is expressed in the form of $\mathrm{C}$. When the sparse matrix is connected, the elements in $\mathrm{C}$ are generated by $\times$ through convo- 
lution with a kernel width of 3 , and the $\mathrm{C} 3$ unit only has a connection relationship with $\mathrm{X} 2$, X3, and X4. These units are called the receptive fields of $\mathrm{C} 3$. In the non-sparse connection, since $\mathrm{c}$ is generated by matrix multiplication, all inputs will affect $\mathrm{C} 3$.

The sparse connection achieves the purpose of reducing the number of weight parameters. There are two advantages to reducing the number of weight parameters: one is to reduce the complexity of the calculation, and the other is that too many connections will cause serious overfitting, and reducing the number of connections can improve the generalization of the model.

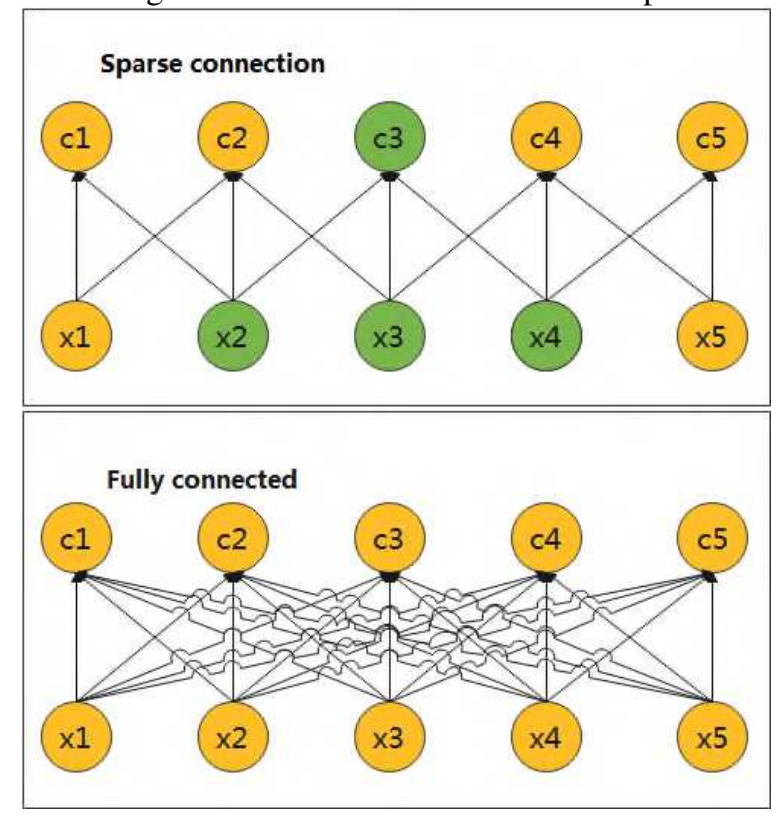

Figure 2 Schematic diagram of sparse connection and full connection

(2) Parameter sharing. In a convolutional neural network, every element of the convolution kernel will act on every position of the pain EEG signal. The parameter sharing mechanism in the convolution operation will also significantly reduce the number of parameters. Compared with each neuron in the fully connected method that needs to learn a separate parameter set, each neuron in the convolution operation only needs to learn a parameter set of the size of the convolution kernel.

Recurrent neural network is a type of recurrent neural network that takes sequence data as input, recursively in the evolution direction of the sequence, and all nodes are connected in a chain. LSTM is called short-term memory network, which is a special kind of RNN, which is memorable and also has the characteristics of parameter sharing. It has certain advantages when learning the nonlinear characteristics of the sequence. The structure of a single unit of LSTM is shown in Figure 3. To summarize the RNN network in one sentence, it is a unit structure that is repeatedly used. The LSTM unit structure contains three gates: input gate, forget gate and output gate.

(1) Input gate: The input gate determinesthe update of the input of the current time step and the internal state of the system state of the previous time step.We look at $h_{t-1}$ and $x_{t}$, and output a number between 0 and 1 for each number in the unit state $C_{t-1} .1$ means "completely retain this condition", and 0 means "completely retain this condition". The principle is shown in formula (4).

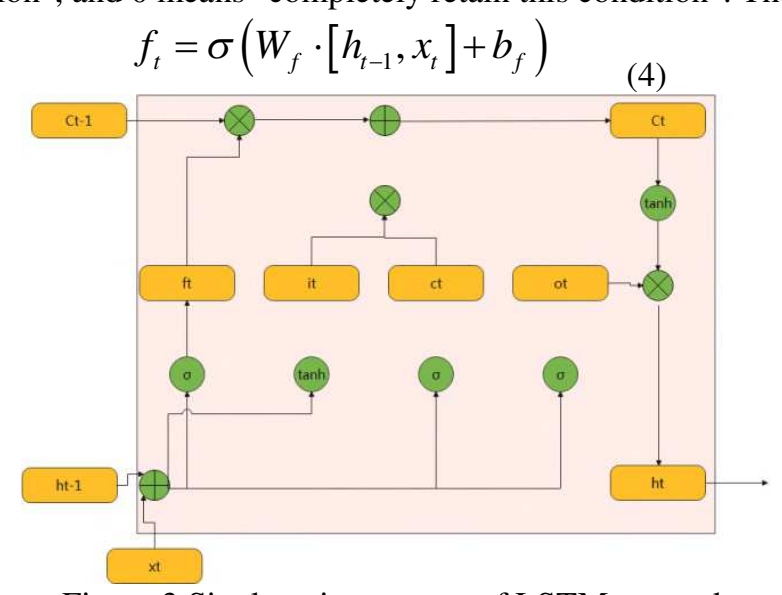

Figure 3 Single unit structure of LSTM network

(2) Forgetting gate: The forgetting gate determines the update of the internal state of the previous time step to the internal state of the current time step. First, the S-shaped layer of the "input gate layer" determines which values will be updated.Next, the activation function uses the tanh function to create a vector of new candidate values $\bar{C}_{t}$, which can be added to the state.The principle is shown in formula (5) and formula (6).

$$
\begin{aligned}
& i_{t}=\sigma\left(W_{i} \cdot\left[h_{t-1}, x_{t}\right]+b_{i}\right) \\
& \bar{C}_{t}=\tanh \left(W_{c} \cdot\left[h_{t-1}, x_{t}\right]+b_{c}\right)
\end{aligned}
$$


Then, the old state $C_{t-1}$ is updated to the new state $C_{t}$, and then $i_{t} * \bar{C}_{t}$ is added.This is a new candidate value. According to the decision, the size of each state value is updated and scaled. The principle is shown in formula (7).

$$
C_{t}=f_{t} * C_{t-1}+i_{t} * \bar{C}_{t}
$$

(3) Output gate: The output gate determines the update of the internal state to the system state. The principle is shown in formulas (8) and (9).

$$
\begin{gathered}
o_{t}=\sigma\left(W_{o} \cdot\left[h_{t-1}, x_{t}\right]+b_{o}\right) \\
h_{t}=o_{t} * \tanh \left(C_{t}\right)
\end{gathered}
$$

To prevent over-fitting, it is usually solved by regularization. The idea is to add a regularization term to the loss function.The loss function on the training set is $J(\omega)$, and $\omega$ represents all the parameters in the entire neural network, including weight $\mathrm{w}$ and bias b. When optimizing, $J(\omega)$ is not directly optimized, but $J(\omega)+\lambda R(\omega)$ is optimized. $R(\omega)$ is the regularization term added to the loss function, the way to solve the norm of the weight parameter, and realize the description of the complexity of the model. a is a value determined in advance, which controls the degree of preference for the weight of the small norm, the larger the preference norm, the smaller the weight.

There are two commonly used functions $R(\omega)$ to describe the complexity of the model. One is $\mathrm{L}_{1}$ regularization. The $\mathrm{L}_{1}$ norm is solved for the weight parameter $\mathrm{w}$, and the calculation formula is shown in (10):

$$
R(\omega)=\|w\|_{1}=\sum_{i}\left|w_{i}\right|
$$

The other is $\mathrm{L}_{2}$ regularization, and the calculation formula is as shown in (11):

$$
R(\omega)=\|w\|_{2}^{2}=\sum_{i}\left|w_{i}\right|^{2}
$$

Minimizing $J(\omega)+\lambda R(\omega)$ means that a balance needs to be found between preference for small norm weights and fitting training data. The basic idea is to reduce the probability that the model fits the noise in the training set by limiting the size of the weight, thereby reducing overfitting.

Both types of regularization have their own advantages and disadvantages. $\mathrm{L}_{1}$ regularization will make the parameters sparser, while $\mathrm{L}_{2}$ regularization will not. The $\mathrm{L}_{1}$ regularization formula is not derivable, and the calculation of the $\mathrm{L}_{2}$ regularization formula is derivable. The calculation of the $\mathrm{L}_{2}$ regularization loss function during optimization is more concise, and the calculation of the $\mathrm{L} 1$ regularization loss function is more complicated.Usually, one is not used in isolation. Sometimes $\mathrm{L}_{1}$ and $\mathrm{L}_{2}$ are used in combination. The formula is as shown in (12):

$$
R(\omega)=\|w\|_{1}=\sum_{i} \alpha\left|w_{i}\right|+(1-\alpha)\left|w_{i}\right|^{2}
$$

Dropout method: The Dropout method is also proposed to prevent overfitting. The specific method is to remove the input layer and the output layer without changing. In the hidden layer in the middle, a part of the neurons is selected in a random manner, and a part of the neurons is "deleted". The specific number of neurons to be randomly deleted in the same layer needs to be set by the network designer (usually 1/2,1/3,1/4, etc.). The above is the Drouout method in the fully connected neural network, which is also similar in the convolutional neural network. The Dropout method is used to randomly delete half of the neurons as shown in Figure 4. The number of neurons with different proportions is set in the networks involved later in this article to prevent overfitting. 


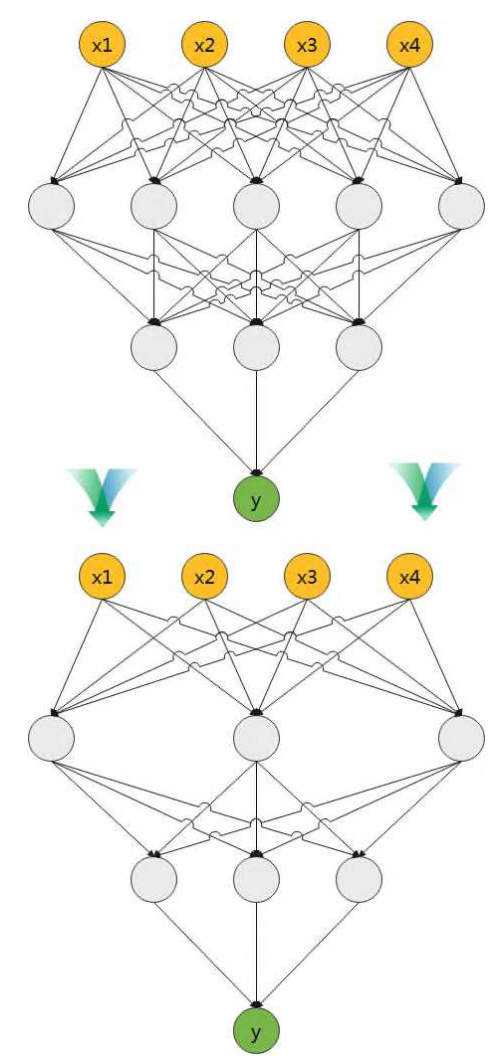

Figure 4 Schematic diagram of the Dropout method randomly deleting half of the neurons

If we just normalize the input data, we can only guarantee that the input layer data distribution is the same, but cannot guarantee that the input data distribution of each layer of the network is the same. Therefore, the middle layer also needs to add normalization.

There are four common normalization methods in deep learning: Batch Normalization, Layer Normalization, Instance Normalization, and Group Normalization. The four normalization calculation processes are almost the same, which can be divided into four steps:

(1) The calculated mean value is shown in formula (13):

$$
\mu_{B}=\frac{1}{m} \sum_{i=1}^{m} x_{i}
$$

(2) The calculated variance formula is shown in (14):

$$
\sigma_{B}^{2}=\frac{1}{m} \sum_{i=1}^{m}\left(x_{i}-\mu_{B}\right)^{2}
$$

(3) The formula with a mean value of 0 and a variance of 1 obtained by the normalization process is shown in (15):

$$
\hat{X}_{t}=\frac{\left(x_{i}-\mu_{B}\right)}{\sqrt{\sigma_{B}^{2}+l}}
$$

(4)Change and reconstruct, restore the distribution that this layer of network needs to learn, the formula is as shown in (16)

$$
y_{i}=\gamma \hat{x}_{i}+\beta=B N_{\gamma, \beta}\left(x_{i}\right)
$$

In actual network training, we can try a variety of normalization methods, and choose the normalization method that suits our own data and network. In the normalization involved in this article, in the preprocessing stage of data input into the network, the first three steps of the above normalization are used to normalize all 3260 EEG data fragments. What is adopted in the network is BatchNormalization method.

\section{Analysis of Industry Convergence Based on Improved Neural Network}

Based on the development trend of the information age, this article is guided by market demand to make up for the defects of the industrial chain by dealing with the contradictions of production lag. The two major industries rely on the enterprise respectively and rely on the intermediary function of the enterprise. It first decomposes the various effective links in their respective industrial chains, and then uses the links to gather together more closely related industrial company groups to form a new industry. The new industry has produced modules with different functions. After the modules are mutually aligned, they form a network, that is, the integration of the two industries is realized. The evolution process of industrial chain-industry convergence can be seen in Figure 5. 


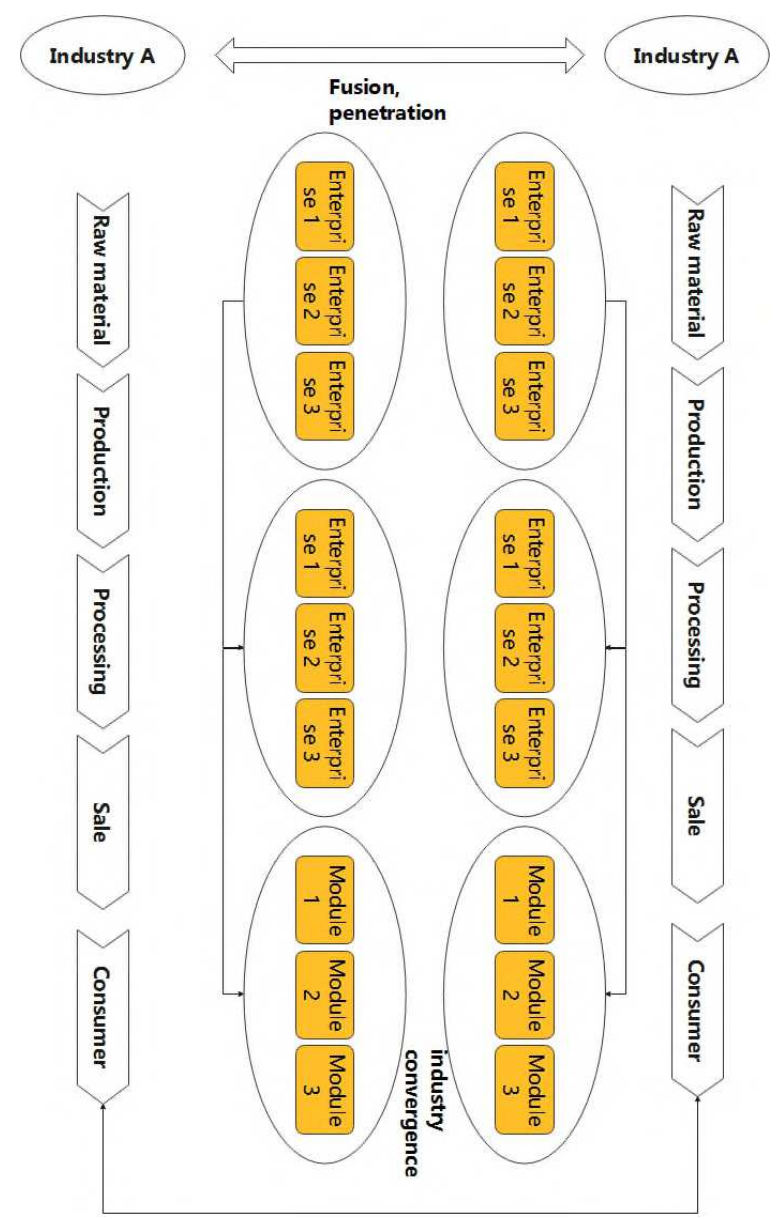

Figure 5 The evolution of industrial chain-industrial convergence

Figure 6 shows an example of industry convergence training based on neural networks. The circled part in the figure is the sample that was misclassified in this iteration, and its weight will be adjusted in the next training, and finally the classification will be completed by means of weighted summation.

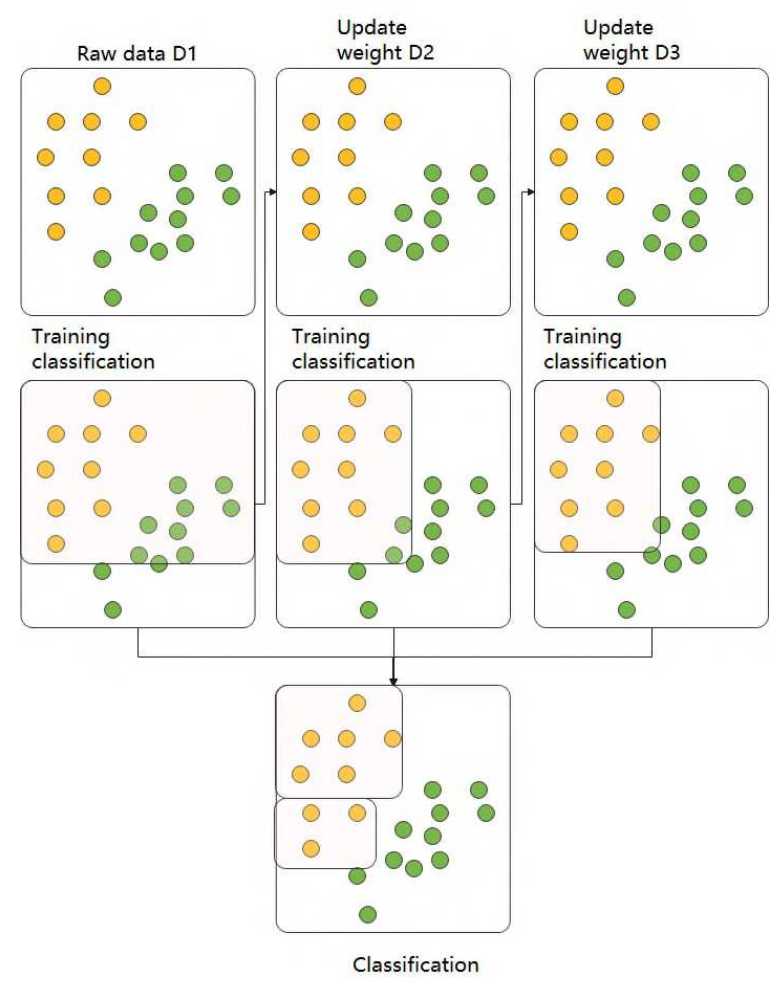

Figure 6 The training process of the industry convergence model

Industry convergence includes technology convergence, business and management convergence, market convergence, product convergence, enterprise convergence, system convergence, and so on. The convergence of different aspects is a manifestation of the process of industry con-vergence. Only by integrating these aspects can the overall meaning of industry convergence be formed, as shown in Figure 7. 


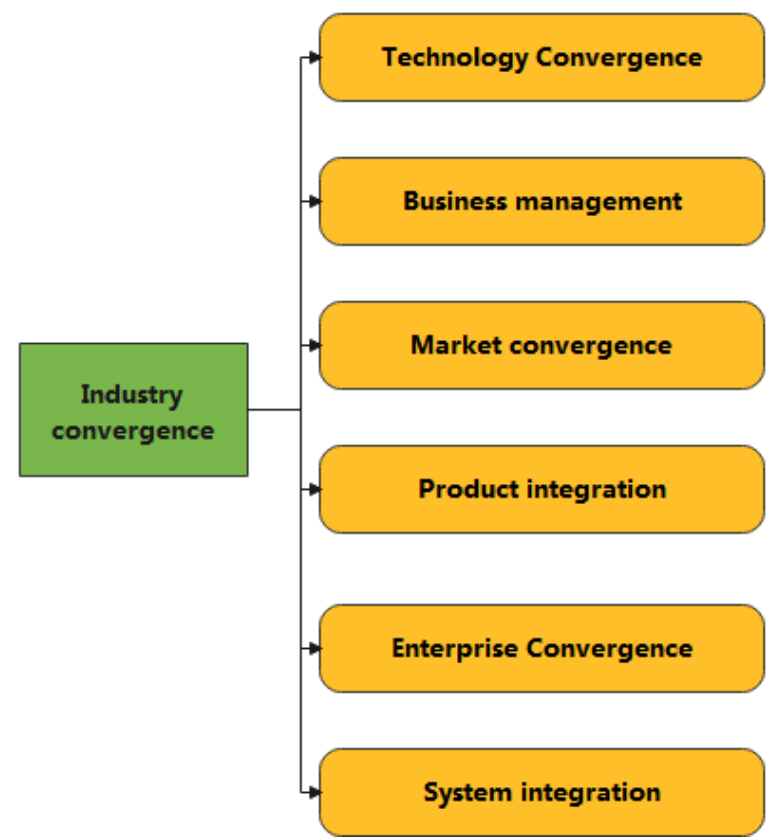

Figure 7 The meaning of industry convergence in the context of digital economy

Industry convergence is divided into four categories: penetration type convergence, complementary type convergence, reorganization type convergence, and alternative type convergence. Penetration typeconvergence often occurs at the junction of high-tech industries and traditional industries, as shown in Figure 8.

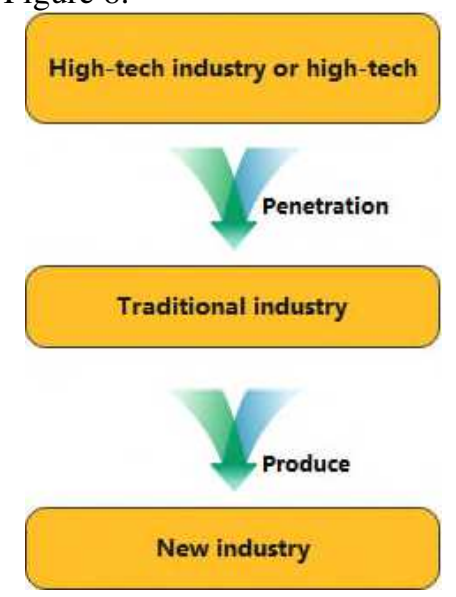

Figure 8 penetration type convergence

Judging from the practice of industry convergence under the Internet economy, it can be seen that there are many factors that promote industry convergence. The convergence motives closely related to the Internet economy are the diffusion effect of technological innovation, the improvement of asset versatility and the expansion of market demand. The motives of convergence that are consistent with the traditional meaning are the relaxation of government regulation, the pressure of enterprises' competition and cooperation, and the pursuit of benefits, as shown in Figure 9. 


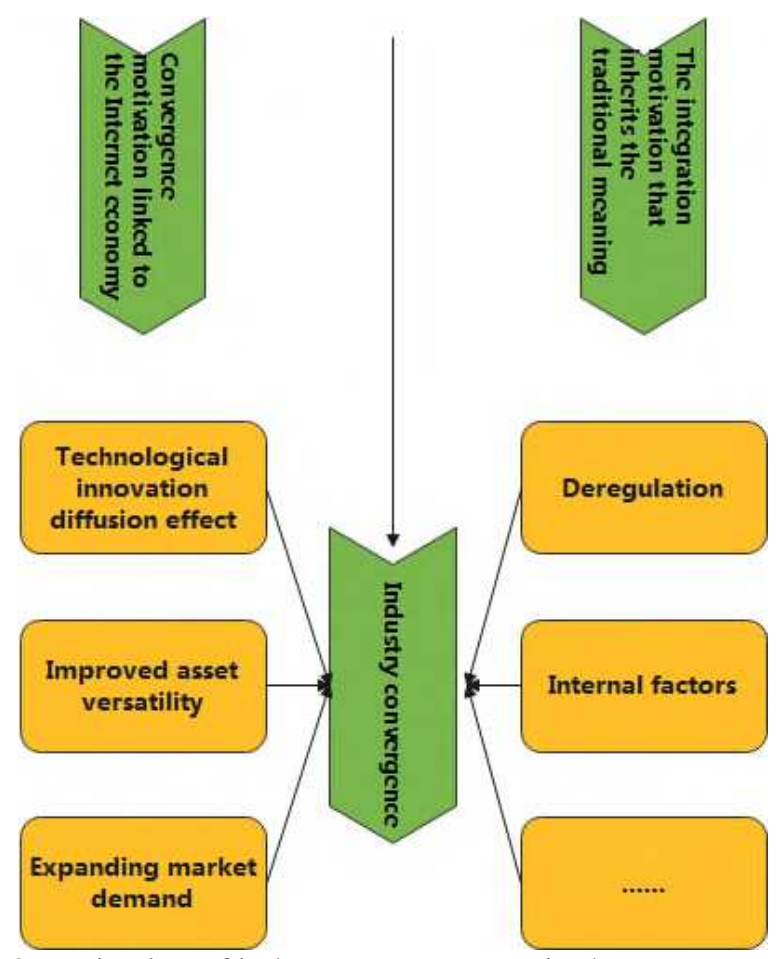

Figure 9 Motivation of industry convergence in the Internet economy

After constructing an industry convergence analysis model based on an improved neural network, the industry convergence of agriculture and information technology is analyzed. This paper conducts experiments through multiple sets of data, and combines the neural network model of this paper to conduct research, and calculates the analysis effect of industry convergence, the prediction effect of industry convergence, and the effect of industry convergence strategy formulation. The results are shown in Table 1 and Figure 10.

Table 1 Performance verification of industrial analysis model based on improved neural network

\begin{tabular}{|c|c|c|c|c|c|c|c|}
\hline Number & $\begin{array}{c}\text { Industrial integration } \\
\text { effect }\end{array}$ & $\begin{array}{l}\text { Industry Convergence } \\
\text { Forecast } \\
\end{array}$ & $\begin{array}{c}\text { Industry Convergence } \\
\text { Policy } \\
\end{array}$ & Number & $\begin{array}{c}\begin{array}{c}\text { Industrial integration } \\
\text { effect }\end{array} \\
\end{array}$ & $\begin{array}{c}\text { Industry Convergence } \\
\text { Forecast } \\
\end{array}$ & $\begin{array}{c}\text { Industry Convergence } \\
\text { Policy } \\
\end{array}$ \\
\hline 1 & 76.12 & 85.71 & 78.71 & 19 & 73.15 & 81.73 & 79.13 \\
\hline 2 & 81.07 & 90.30 & 92.12 & 20 & 83.26 & 82.40 & 74.25 \\
\hline 3 & 79.54 & 77.53 & 86.54 & 21 & 84.35 & 89.61 & 83.61 \\
\hline 4 & 72.21 & 86.46 & 79.04 & 22 & 76.34 & 79.29 & 72.19 \\
\hline 5 & 87.15 & 79.77 & 76.20 & 23 & 77.95 & 77.04 & 85.47 \\
\hline 6 & 88.90 & 74.64 & 85.49 & 24 & 71.29 & 82.38 & 87.68 \\
\hline 7 & 78.46 & 78.19 & 89.19 & 25 & 72.76 & 86.02 & 89.50 \\
\hline 8 & 84.89 & 75.65 & 90.38 & 26 & 85.55 & 90.26 & 87.99 \\
\hline 9 & 76.29 & 79.30 & 81.72 & 27 & 78.92 & 90.71 & 85.13 \\
\hline 10 & 85.30 & 70.82 & 81.87 & 28 & 84.13 & 75.34 & 85.06 \\
\hline 11 & 76.56 & 79.25 & 91.94 & 29 & 73.79 & 76.17 & 79.03 \\
\hline 12 & 76.03 & 78.65 & 76.45 & 30 & 74.53 & 83.37 & 86.92 \\
\hline 13 & 88.50 & 83.01 & 83.57 & 31 & 79.79 & 83.68 & 89.97 \\
\hline 14 & 80.18 & 83.09 & 80.79 & 32 & 77.36 & 73.74 & 72.40 \\
\hline 15 & 73.26 & 90.14 & 72.09 & 33 & 85.90 & 87.22 & 83.69 \\
\hline 16 & 77.44 & 85.05 & 84.56 & 34 & 85.79 & 80.95 & 80.33 \\
\hline 17 & 81.98 & 85.88 & 75.72 & 35 & 82.43 & 89.20 & 76.49 \\
\hline 18 & 73.50 & 71.58 & 81.94 & 36 & 88.51 & 84.91 & 85.75 \\
\hline
\end{tabular}




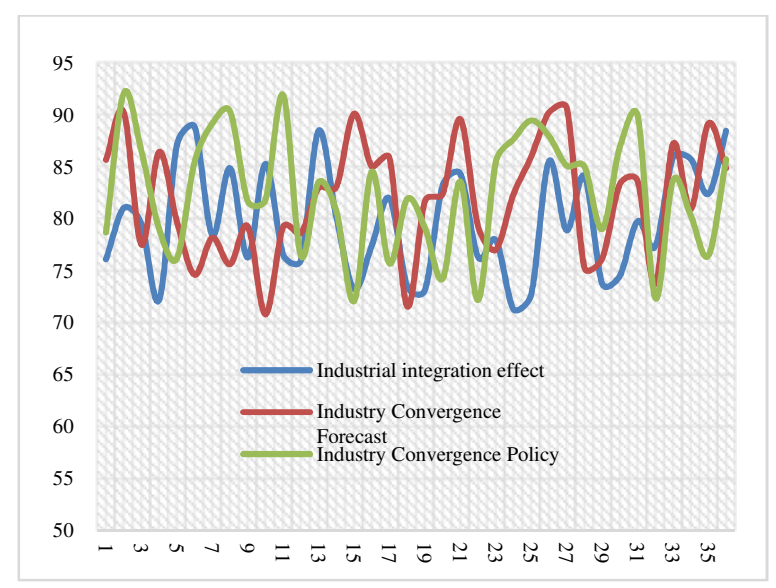

Figure 10 Statistical diagram of experimental research results

From the above research, it can be seen that the industry convergence analysis model based on the improved neural network constructed in this article can effectively improve the analysis effect of industry convergence, and has a certain promotion effect on subsequent industry convergence research.

\section{Conclusion}

In addition to product convergence, industry convergence also involves other aspects of integration, such as technological convergence, deregulation, and system convergence. It is a complex economic phenomenon that integrates multiple aspects.In the current process of economic development, the rapid rise of high-tech industries has become an important driving force for economic progress. In the context of the convergence and growth of the high-tech industry, this article combines the improved neural network algorithm to construct the industry convergence analysis model. Moreover, based on the development trend of the information age, this article is guided by market demand to make up for the defects of the industrial chain by dealing with the contradictions of production lag. The two major industries rely on the enterprise respectively and rely on the intermediary function of the enterprise. This paper first decomposes the effective links in their respective industrial chains, analyzes the industry convergence with the improved neural network algorithm, proposes the corresponding system function modules, and verifies the reliability of the model in this paper through experimental research.

\section{Declarations}

Funding: This work is supported by the Philosophy and Social Science Planning Project of Fujian Province: Research on the Evolution Mechanism of Collaborative Innovation System of Strategic Emerging Industries in Fujian Province(Project No.: FJ2018MGCZ011), and the High-level talent scientific research start-up fund project of Fuzhou University of International Studies and Trade: Research on Evolution Mechanism of Chinese Strategic Emerging Industries Collaborative Innovation System(Project No.: FWB19002).

Conflicts of interests: None

Availability of data and material: Not applicable.

Code availability: Not applicable.

Authors' Contributions: Nan Ma designed the model, collected dataset, performed the analysis, validated the results, written and reviewed the manuscript.

\section{Compliance with Ethical Standards}

Disclosure of potential conflicts of interest: No potential conflicts.

Research involving Human Participants and/or Animals: No involvement of humans or animals.

Informed consent: Not Applicable.

\section{References}

[1]. Akhmetshin E M, Kolpak E P, Sulimova E A, et al. Clustering as a criterion for the success of modern industrial enterprises[J]. International Journal of Applied Business and Economic Research, 2017, 15(23): 221-231.

[2]. Belso-Martinez J A, Diez-Vial I, Lopez-Sanchez M J, et al. The brokerage role of supporting organizations inside clusters: how does it work?[J]. European Planning Studies, 2018, 26(4): 706-725.

[3]. Chain C P, Santos A C, Castro L G, et al. Bibliometric analysis of the quantitative methods applied to the measurement of industrial clusters[J]. Journal of Economic Surveys, 2019, 33(1): 60-84.

[4]. Chandrashekar D, BalaSubrahmanya M H. Exploring the factors of cluster linkages that influence innovation performance of firms in a cluster[J]. Economics of Innovation and new Technology, 2019, 28(1): 1-22.

[5]. EL WAATMANI A. Industrial clusters and promotion of CSR: the case of developing countries[J]. Journal of Academic Fi- 
nance, 2018, 9(2): 69-80.

[6]. Götz M, Jankowska B. Clusters and Industry 4.0-do they fit together?[J]. European Planning Studies, 2017, 25(9): 16331653.

[7]. Guowu L, Bai G A O. Globalization and Domestic Coping Strategies: The Development of China's Industrial Clusters[J]. İstanbul University Journal of Sociology, 2020, 40(2): 625-647.

[8]. Hou B, Hong J, Wang H, et al. Academia-industry collaboration, government funding and innovation efficiency in Chinese industrial enterprises[J]. Technology Analysis \& Strategic Management, 2019, 31(6): 692-706.

[9]. Isaksen A, Trippl M. Innovation in space: the mosaic of regional innovation patterns[J]. Oxford Review of Economic Policy, 2017, 33(1): 122-140.

[10]. Jones C, Pimdee P. Innovative ideas: Thailand 4.0 and the fourth industrial revolution[J]. Asian International Journal of Social Sciences, 2017, 17(1): 4-35.

[11]. Kuksa I, Shtuler I, Orlova-Kurilova O, et al. Innovation cluster as a mechanism for ensuring the enterprises interaction in the innovation sphere[J]. Management Theory and Studies for Rural Business and Infrastructure Development, 2019, 41(4): 487500.

[12]. Lazzeretti L, Capone F, Caloffi A, et al. Rethinking clusters. Towards a new research agenda for cluster research[J]. European Planning Studies, 2019, 27(10): 1879-1903.

[13]. Liu Z, Chen X, Chu J, et al. Industrial development environment and innovation efficiency of high-tech industry: Analysis based on the framework of innovation systems[J]. Technology Analysis \& Strategic Management, 2018, 30(4): 434-446.

[14]. Moradi M, ZandiPaak R. The Effect of Companies' Participation or Non-Participation in the Creation of Industrial Clusters on Their Innovation Performance with an Emphasis on the Mediatory Role of Knowledge Management[J]. Innovation Management Journal, 2023, 5(1): 1-28.

[15]. Novotná J, Novotný L. Industrial clusters in a post-socialist country: The case of the wine industry in Slovakia[J]. Moravian Geographical Reports, 2019, 27(2): 62-78.

[16]. Schot J, Steinmueller W E. Three frames for innovation policy: R\&D, systems of innovation and transformative change[J]. Research Policy, 2018, 47(9): 1554-1567.

[17]. Sosnovskikh S. Industrial clusters in Russia: The development of special economic zones and industrial parks[J]. Russian Journal of Economics, 2017, 3(2): 174-199.

[18]. Sozinova A A, Okhrimenko O I, Goloshchapova L V, et al. Industrial and innovation clusters: development in Russia[J]. International Journal of Applied Business and Economic Research, 2017, 15(11): 111-118.

[19]. Stavroulakis P J, Papadimitriou S. Situation analysis forecasting: the case of European maritime clusters[J]. Maritime Policy \& Management, 2017, 44(6): 779-789.

[20]. Tödtling F, Trippl M. Regional innovation policies for new path development-beyond neo-liberal and traditional systemic views[J]. European Planning Studies, 2018, 26(9): 1779-1795.

[21]. Turkina E, Van Assche A. Global connectedness and local innovation in industrial clusters[J]. Journal of International Business Studies, 2018, 49(6): 706-728.

[22]. Ugalde Hernández O. Local industrial agglomerations vis-à-vis global competitive networks: marshallian notions of clusters, innovation and territorial development[J]. Economía y Sociedad, 2020, 25(57): 42-62.

[23]. Žižka M, Valentová V, Pelloneová N, et al. The effect of clusters on the innovation performance of enterprises: traditional vs new industries[J]. Entrepreneurship and Sustainability Issues, 2018, 5(4): 780-794.

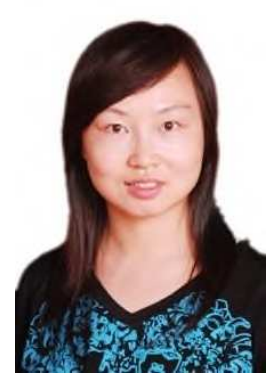

Nan Ma (1982- ), female, doctor, associate professor of Fuzhou University of International Studies and Trade 